\title{
Combining the Pilot Point and Gradual Deformation Methods for Calibrating Permeability Models to Dynamic Data
}

\author{
M. Le Ravalec-Dupin ${ }^{1}$ and L.Y. Hu' \\ 1 Institut français du pétrole, IFP, Reservoir engineering department, 1 et 4, avenue de Bois-Préau, 92852 Rueil-Malmaison Cedex - France \\ email: mickaele.le-ravalec@ifp.fr - l-ying.hu@ifp.fr
}

\begin{abstract}
Résumé - Utilisation combinée de la méthode des points pilotes et de la méthode de déformation graduelle pour contraindre des modèles de perméabilité par des données dynamiques - On se concentre sur des techniques de paramétrage développées dans le cadre de la modélisation inverse de champs de perméabilité. Deux contributions essentielles sont la méthode des points pilotes et la méthode de déformation graduelle. Ces techniques ont été mises au point pour réduire le nombre de paramètres et pour assurer la préservation de la structure spatiale malgré les perturbations. La méthode des points pilotes pose problème sur deux points : des valeurs extrêmes et aberrantes peuvent être attribuées aux points pilotes et les corrélations spatiales entre les points pilotes sont négligées. Pour outrepasser ces difficultés, nous proposons une nouvelle technique de paramétrage, la méthode des points pilotes graduels. Elle s'organise comme la méthode originale des points pilotes, hormis le fait que les valeurs des points pilotes sont ici modifiées par l'intermédiaire des paramètres de déformation graduelle et non directement par l'optimiseur. Contrairement aux points pilotes usuels, les points pilotes graduels ne prennent pas de valeurs extrêmes. En outre, lorsque tous les points pilotes graduels sont perturbés à partir d'un unique paramètre de déformation, les corrélations spatiales entre les points pilotes sont prises en compte. Aussi de nombreux points pilotes peuvent-ils être placés sur le modèle de perméabilité à modifier sans se soucier des positions sélectionnées. Les points pilotes graduels sont utilisés pour modifier un modèle localement ou globalement. L'expérience numérique développée pour illustrer l'intérêt de la méthode des points pilotes graduels est construite suivant deux étapes. Tout d'abord, le modèle de perméabilité est modifié dans sa globalité à l'aide de la méthode de déformation graduelle, puis des améliorations y sont localement apportées en utilisant la méthode des points pilotes graduels.
\end{abstract}

\footnotetext{
Abstract - Combining the Pilot Point and Gradual Deformation Methods for Calibrating Permeability Models to Dynamic Data - We focus on specific parameterization techniques developed in inverse stochastic modeling for determining permeability fields from dynamic data using a reduced number of parameters. Two major contributions are the pilot point method and the gradual deformation method. They were designed to reduce the number of parameters and to respect the inferred spatial structure. Weaknesses have been revealed for the pilot point method: pilot points can be assigned unreasonably extreme values and possible correlations among the pilot points are neglected. To bypass these limitations, a new approach, called the gradual pilot point method, is suggested. It follows the basic workflow of the pilot point method, but the pilot point values are not driven by the optimization procedure. Intermediate gradual deformation parameters are introduced which govern the pilot point values. Compared to the original pilot point method, the gradual pilot point method does not produce extreme
} 
variations. Moreover, when the whole set of pilot points is modified simultaneously from a single deformation parameter, the correlations among the pilot points are accounted for. Thus, many pilot points can be placed on the permeability field, whatever their locations. They can produce local and global deformation. The performed numerical experiments show that a two-step approach for calibrating permeability fields is useful. First, the gradual deformation method is used to globally deform the permeability fields. Once the permeability fields have been globally improved, they can be locally refined using the gradual pilot point method.

\section{INTRODUCTION}

Identifying a permeability or a porosity field from the available production data (pressures, flow rates, water cuts, etc.) and the collected 4D-seismic attributes is an important problem in reservoir engineering and hydrology. Given a flow model, it consists in approximating a subsurface reservoir by a grid of permeability or porosity values for which the simulated flow response replicates the observed production and 4D-seismic data. The determination of the permeability or porosity values can be performed by linearizing the fluid flow equation. Such an approach is valid as long as the permeability field is not too heterogeneous. An alternative is to invert the production data based on an optimization process. The problem boils down to minimizing an objective function which quantifies the mismatch between the measured data and the corresponding simulated data. For fluid flow simulation, it is common to handle millions of grid cells, with each grid cell having a permeability and a porosity value. In this case, the purpose of the optimization process is to identify millions of unknowns from the available data.

The difficulty in analyzing subsurface flow is mainly due to the heterogeneity of the geological formations. This complexity is often described by a random function with a spatial structure (e.g., variogram) inferred from the measured permeability or porosity data. This feature motivated the development of specific parameterization techniques which ensures the preservation of spatial structure and which decrease the number of parameters. A first geostatistical parameterization technique was introduced by de Marsily (1978): it is often referred to as the pilot point method or the master location method. This technique was extended later by RamaRao et al. (1995) and Gomez-Hernandez et al . (1997) to the calibration of permeability fields to pressure data. Recently, a second geostatistical parameterization technique was suggested by $\mathrm{Hu}(2000 \mathrm{a})$ : the gradual deformation method. Both techniques provide means to modify permeability and porosity fields from a limited number of parameters while preserving the spatial variability, which is a major improvement compared to other existing parameterization techniques.

In this paper, we review the major contributions developed for determining permeability fields from production data. First, we recap how permeability fields are built and which kind of data is integrated in the objective function to be minimized. Secondly, we focus on the existing geostatisti- cal parameterization techniques, that is the pilot point method and the gradual deformation method. Then, we suggest mixing both of them to derive a new version of the pilot point method, called the gradual pilot point method. This last approach overcomes the limitations often pointed out for the original pilot point method. The last section focuses on a numerical example, which stresses the interest of the proposed parameterization technique.

\section{INVERSE PROBLEM}

Production data and 4D-seismic attributes are called dynamic data since they change with time due to fluid displacements. Here, permeability fields are considered as realizations of a random function. They are used to populate grids with a finite number of cells. As a result, the whole paper focuses on finite permeability realizations. This random function is defined from a variogram, which characterizes the distribution of permeability (or porosity) values in space. Identifying a permeability field from dynamic data is an inverse problem. When simulating multi-phase flow, the relationship between the petrophysical properties and the dynamic answers is highly non-linear. Thus, the method to find a solution must be iterative. A solution can be reached through an optimization procedure. In such a case, an objective function quantifying the suitability of the permeability field has to be defined and minimized. The first difficulty is about the objective function definition, the second one being about the minimization process.

Early approaches placed the emphasis on providing a good fit only between the simulated and the measured production data. Basically, a log-permeability field $\mathbf{y}$ is proposed as a starting guess. Then, the production answers are computed through a fluid flow simulation. The suitability of the proposed log-permeability field is given by the objective function:

$$
J(y)=\sum_{i} w_{i}\left(d_{\text {sim }_{i}}-d_{o b s_{i}}\right)^{2}
$$

Summation is over the total number of dynamic data. $w_{i}$ are weighting coefficients, $d_{o b s i}$ are the collected dynamic data, and $d_{s i m i}$ are the corresponding simulated answers. $g$ is the operator from the space of unconditional log-permeability 
fields to the space of production data: $\mathbf{d}_{s i m}=g(\mathbf{y})$. After analyzing the computed data, the log-permeability field is adjusted until it reproduces the dynamic data as well as possible. As Equation 1 does not account for correlations among the permeability values, the final calibrated permeability field can exhibit any kind of spatial structure, which is not suitable.

The actual log-permeability field should be the minimizer of the objective function. However, when solving inverse problems, a difficulty known as ill-posedness may arise (e.g., Sun, 1994). A well-posed problem must fulfill the following requirements:

- a solution must exist;

- the solution must be unique;

- the solution must continuously depend on the data.

If one of these criteria is not fulfilled, the problem is illposed. The third criterion is often difficult to respect. Adding small perturbations in the data may force large errors in the solution. To make the problem well-posed or at least, "less" ill-posed, one refers to regularization. If regularization is properly performed, it should reduce the risk of introducing large errors in the solution and exclude the possibility of producing non-physical solutions. Two regularization processes are usually listed.

The first one is based upon the addition of extra information into the objective function:

$$
J(y)=\sum_{i} w_{i}\left(d_{s i m_{i}}-d_{o b s_{i}}\right)^{2}+\alpha \sum_{j} v_{j}\left(y_{j}-y_{o_{j}}\right)^{2}
$$

Index $i$ refers to the available dynamic data while index $j$ refers to the parameters of the model. Vector $\mathbf{y}$ is the current estimate of the log-permeability field, vector $\mathbf{y}_{o}$ is its prior estimate and vector $\boldsymbol{v}$ gathers weighting coefficients. Subscript $i$ lists the vector components. $\alpha$ is a regularization coefficient. The second right-hand side term or regularization term prevents the log-permeability field $\mathbf{y}$ from departing too far from the prior estimate. For zero $\alpha$, the regularization term vanishes and the problem may be ill-posed. The problem boils down to the previous case when there is only dynamic data in the objective function. For infinite $\alpha$, the problem is well posed, but the observed data have no impact on the solution. The greatest problem is to find an appropriate value for $\alpha$. Neuman (1973) showed that adding prior information in the objective function maintains the log-permeability field close to the prior estimate as desired, but also stabilizes the optimization process. A more general framework for integrating prior information is given by the inverse problem theory (Tarantola, 1987):

$$
\begin{aligned}
J(y) & =\frac{1}{2}\left(g(y)-d_{o b s}\right)^{t} C_{D}^{-1}\left(g(y)-d_{o b s}\right) \\
& +\frac{1}{2}\left(y-y_{o}\right)^{t} C_{Y}^{-1}\left(y-y_{o}\right)
\end{aligned}
$$

The first term on the right-hand side is the likelihood constraint and measures the mismatch between the simulated and the observed data. The second one is the prior constraint and evaluates the discrepancy between $\log$-permeability field $\mathbf{y}$ and the prior estimate $\mathbf{y}_{o}$. Covariance matrix $\mathbf{C}_{D}$ quantifies the experimental and theoretical uncertainties and covariance matrix $\mathbf{C}_{Y}$ characterizes the uncertainties in $\mathbf{y}_{o}$. Minimizing this equation yields a solution $\mathbf{y}$ so that $g(\mathbf{y})$ is close to $\mathbf{d}_{o b s}$ and $\mathbf{y}$ is close to $\mathbf{y}_{o}$. Computing such an objective function may be difficult because of the two covariance matrices to invert. $\mathbf{C}_{D}$ is assumed to be diagonal: computing its inverse is straightforward. Discussing this hypothesis is beyond the scope of this paper. Conversely, determining $\mathbf{C}_{Y}{ }^{-1}$ may be very difficult, essentially because of the dimension of $\mathbf{y}$. An example of application is provided in seismic inversion. Brac et al. (1988) and Tonellot (1999) proposed to add geological information into the objective function through the prior term. $\mathbf{C}_{Y}$ was considered to be a function of the range, the variance and the variogram type. The range and the variance were defined from the spatial structure. The variogram was assumed to be exponential to make computations tractable.

The regularization process can be also envisioned from a different point of view. Instead of adding information into the objective function, one may narrow the space wherein the solution is searched. To successfully restrict the parameter space, some knowledge is required about the nature of the solution. In reservoir engineering, one often assumes some geological continuity, which characterizes the spatial distribution of porosity and permeability in the reservoir. The zonation approach (Stallman, 1956) was developed with this idea in mind. This comprises grouping grid cells to create subregions with constant permeability values. Optimization is then performed by adjusting these values. Unfortunately, the spatial structure handled by the zonation method keeps rough. In this paper, we give attention to more sophisticated geostatistically-based parameterization techniques, which also add restriction to the solution space: the pilot point method and the gradual deformation method. These methods allow for perturbing log-permeability (or porosity) fields from a few parameters while preserving the spatial variability. Therefore, the objective function is reduced to the data mismatch term:

$$
J(y)=\frac{1}{2}\left(g(y)-d_{o b s}\right)^{t} C_{D}^{-1}\left(g(y)-d_{o b s}\right)
$$

In such a case, it is not necessary to invert $\mathbf{C}_{Y}$. The prior constraint, that is the spatial structure, is accounted for through the parameterization itself. This feature is discussed in the following sections.

\section{PILOT POINT METHOD}

The pilot point method can be used to calibrate permeability fields to dynamic data (RamaRao et al., 1995; 


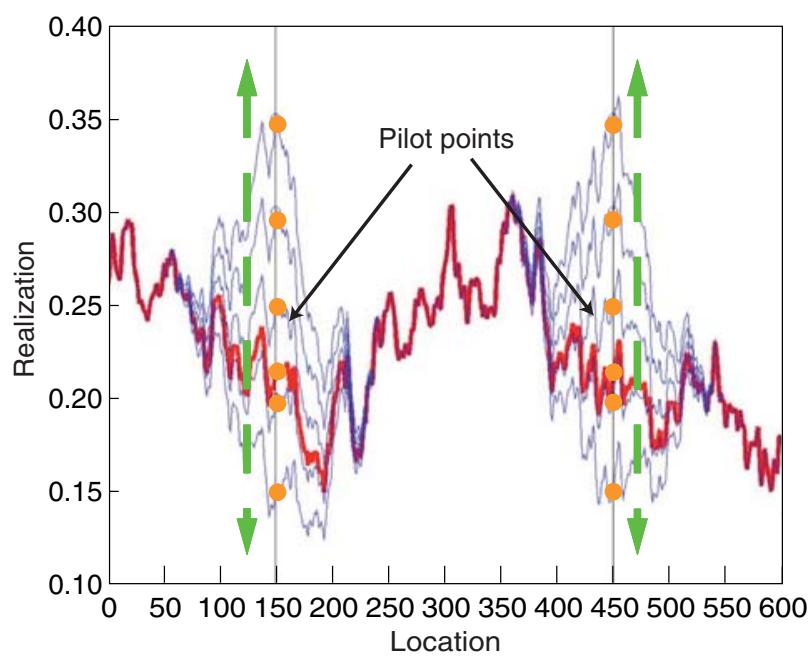

Figure 1

Applying the pilot point method to change the values of points located in 150 and 450 for a one dimensional realization of a normal random function.
Gomez-Hernandez et al.; 1997). At this point, the idea is to identify a log-permeability field from production or 4D-seismic data using the pilot point method. Log-permeability means that we handle the logarithm of permeability instead of permeability itself. The reason is that the distribution of permeability is lognormal. Thus, the distribution of logpermeability is normal.

\subsection{Pilot Point}

Let us consider a randomly drawn log-permeability field $\mathbf{y}$ discretized over a grid: it does not respect any permeability measurement except luckily. A pilot point is a grid cell whose log-permeability value may be modified (Fig. 1). The resulting perturbation is propagated to the whole logpermeability field by conditional kriging (e.g., Goovaerts, 1997; Chilès and Delfiner, 1999):

$$
y_{c}(x)=y_{d K}(x)+\left[y(x)-y_{K}(x)\right]
$$
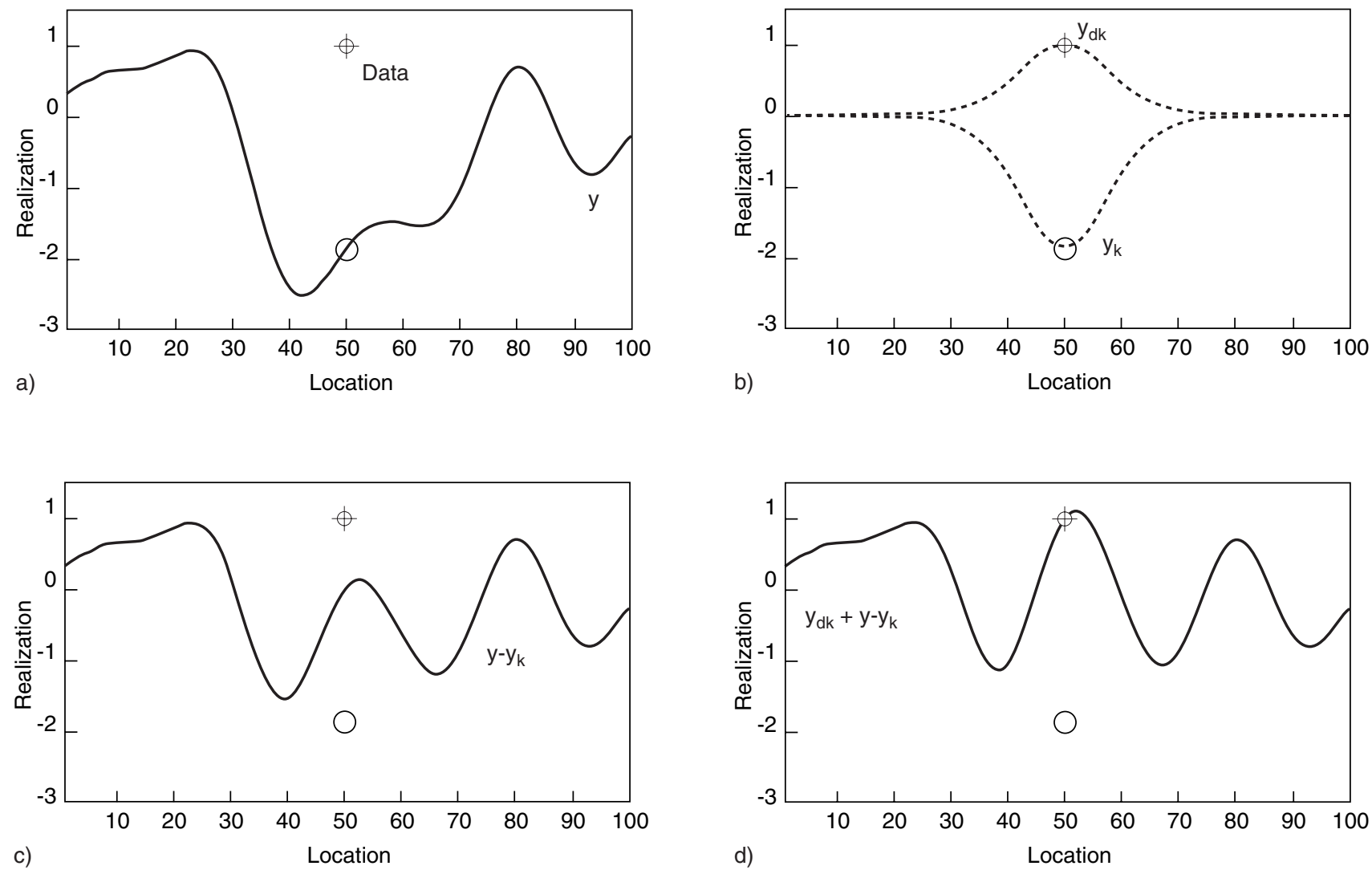

Figure 2

a) Unconditional log-permability field (y) and log-data.

b) Kriging estimate built from the log-data $\left(\mathbf{y}_{\mathrm{dk}}\right)$ and kriging estimate built from the unconditional value simulated at the data location $\left(\mathbf{y}_{\mathrm{k}}\right)$.

c) Unconditional log-permeability field minus the kriging estimate built from the unconditional value simulated at data location.

d) Constrained realization. 
$\mathbf{y}_{c}$ is the log-permeability field constrained to the available log-permeability data, but also to the values attributed to the pilot points. $\mathbf{y}_{d K}$ is the kriging estimate of the log-permeability measurement and the pilot point values. $\mathbf{y}_{K}$ is the kriging estimate of the $\mathbf{y}$ values simulated at the log-permeability data locations plus the pilot point locations. A one dimensional example is shown in Figure 2 to illustrate how Equation 5 is used. To apply this relationship, one must know the mean and covariance of $\mathbf{y}$ as well as the locations and values of the measurements. The pilot point is considered just as a data, although its value can change during an optimization process. Such an approach ensures, under some constraints regarding the lag between pilot points, that the modified log-permeability field still honors the same variability or covariance model as the starting log-permeability field. This point is further discussed in the following section. Figure 1 shows realizations of a normal random function obtained when varying the values of two pilot points.

\subsection{Optimization with Pilot Points}

Optimization processes based upon the pilot point method are built as described below. The pilot point values are regarded as unknowns, which are progressively adjusted by the optimizer to minimize the objective function. We recall that the objective function is reduced to the data mismatch term as given by Equation 4 .

Given a stochastic function, a log-permeability field $\mathbf{y}$ is randomly drawn to populate the grid. Once a few grid cells have been selected to be pilot points, their $\mathbf{y}$ values are used as starting values by the optimizer. Then, the log-permeability field is constrained to the log-permeability data and the pilot point values. A fluid flow simulation is run with the resulting conditional field. The computed production answers are compared to the collected dynamic data to estimate the objective function. If this objective function is small enough, the search process is stopped. If not, the pilot point values are modified and the process is iterated. Describing the multiplicity of the pilot point versions is beyond our scope. A more detailed review is available in de Marsily et al. (2000).

The pilot point method allows for decreasing the number of parameters and for honoring the prior constraint provided the pilot point locations are not too close to each other: the pilot point values are modified without accounting for possible correlations. It is also clear that the pilot point method may be subject to numerical artefacts. RamaRao et al. (1995) noticed that pilot points may be attributed unreasonably extreme values to force the fluid flow simulation to reproduce the measured dynamic data.

\section{GRADUAL DEFORMATION METHOD}

The gradual deformation method was initially developed for gradually changing Gaussian stochastic reservoirs models while preserving their spatial variability (Hu, 2000a). Then, it was extended to non Gaussian reservoir models simulated from sequential indicator (Hu et al., 1999) and Boolean $(\mathrm{Hu}$, 2000b; Le Ravalec-Dupin and $\mathrm{Hu}, 2004$ ) algorithms. The gradual deformation method is a stochastic parameterization technique just as the pilot point method, which allows for narrowing the search space.

\subsection{Global Deformation}

The simplest gradual deformation scheme consists in combining two independent Gaussian random functions $Y_{1}$ and $Y_{2}$ with mean $y_{o}$ and identical covariances:

$$
\left[Y(\theta)-y_{o}\right]=\left[Y_{1}-y_{o}\right] \cos (\theta)+\left[Y_{2}-y_{o}\right] \sin (\theta)
$$

$\theta$ is a deformation parameter. This relation ensures that $Y(\theta)$ is also a random Gaussian function with the same mean and the same covariance as $Y_{1}$ and $Y_{2}$, whatever the value of the deformation parameter. Given two independent realizations $\mathbf{y}_{1}$ and $\mathbf{y}_{2}$ of $Y_{1}$ and $Y_{2}$, a continuous realization chain $\mathbf{y}$ can be described varying the deformation parameter:

$$
\left[y(\theta)-y_{o}\right]=\left[y_{1}-y_{o}\right] \cos (\theta)+\left[y_{2}-y_{o}\right] \sin (\theta)
$$

$\mathbf{y}_{1}$ is considered as our initial realization. $\mathbf{y}_{2}$ is just a randomly drawn independent realization. Varying the deformation parameter induces the continuous deformation of realization $\mathbf{y}_{1}$ (Fig. 3). When $\theta$ is zero, $\mathbf{y}=\mathbf{y}_{1}$. When it is $\pi / 2$, $\mathbf{y}=\mathbf{y}_{2}$. Applying Equation 7 leads to the gradual deformation of the whole initial realization. As a result, the deformation is said global.

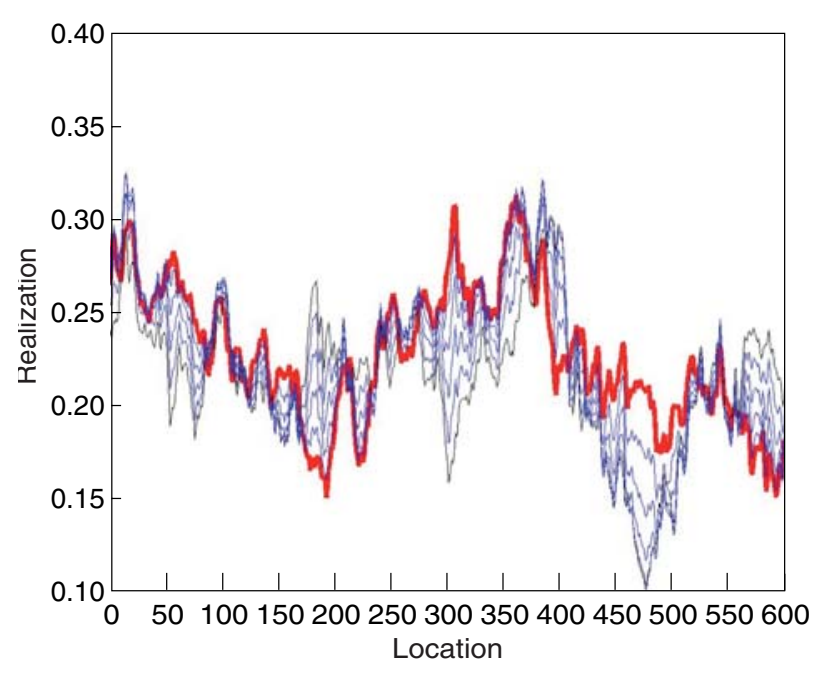

Figure 3

Applying the global gradual deformation to modify the same starting one dimensional realization as in Figure 1. 
The gradual deformation principle can be extended to the combination of more than two independent realizations (Roggero and $\mathrm{Hu}, 1998$ ). In this case, the number of deformation parameters equals the number of complementary realizations added to the starting one.

\subsection{Local Deformation}

The building of a $\mathbf{y}$ log-permeability field calls for the generation of random numbers to populate all of the grid cells. These ones can be transformed into independent normal deviates: they provide a Gaussian white noise, termed $\mathbf{z}$, of mean zero and variance 1 .

The Gaussian white noise $\mathbf{z}$ is used to produce a $\mathbf{y} \log$ permeability field from some stochastic simulation technique (e.g., Le Ravalec et al., 2000). For instance, the Cholesky decomposition method yields a $\mathbf{y} \log$-permeability field from $\mathbf{y}=\mathbf{m}+\mathbf{L z} . \mathbf{m}$ is the mean vector and $\mathbf{L}$ is a lower triangular matrix built from the covariance matrix of $\mathbf{y}$ : $\mathbf{C}=\mathbf{L} \mathbf{L}^{\mathrm{t}}$. The main difference between $\mathbf{y}$ and $\mathbf{z}$ relies on the spatial structure: $\mathbf{y}$ is a realization with a continuous spatial structure and $\mathbf{z}$ exhibits a chaotic behavior. This difference is essential when handling local gradual deformation. When you locally apply a gradual deformation process, you induce a discontinuity between the modified zone and the non modified one. If you locally apply the gradual deformation process to $\mathbf{y}$, you severely perturb its continuous spatial structure, which must be avoided. If $\mathbf{z}$ is locally changed from the gradual deformation method, it is still chaotic and the spatial continuity of the resulting $\mathbf{y}$ realization is preserved.

Therefore, we apply the gradual deformation method to $\mathbf{z}$ instead of $\mathbf{y}$. We can select a group of grid cells and modify their normal deviates through the following formulation:

$$
\left[Y(\theta)-y_{o}\right]=\left[Y_{1}-y_{o}\right] \cos (\theta)+\left[Y_{2}-y_{o}\right] \sin (\theta)
$$

$\mathbf{z}_{1}$ and $\mathbf{z}_{2}$ yield realizations $\mathbf{y}_{1}$ and $\mathbf{y}_{2}$, respectively. The superscript "group" stands for "selected grid cells". When applying Equation 8 to the selected normal deviates only (Hu, 2000a; Le Ravalec-Dupin et al., 2001), the y realization, built from $\mathbf{z}$, is modified in the corresponding selected grid cells. In fact, the neighboring grid cells (that is, closer than one correlation length) are also impacted by the deformation process. The deformation is said to be local.

A degenerate case of the local gradual deformation consists in applying the gradual deformation process to the normal deviate of a single point. The group of cells reduced to one cell. Thus, the modified point gets kind of a pilot point. However, in these conditions, the magnitude of the resulting deformation is much smaller than what was observed when using the pilot point method (Figs. 1 and 4).

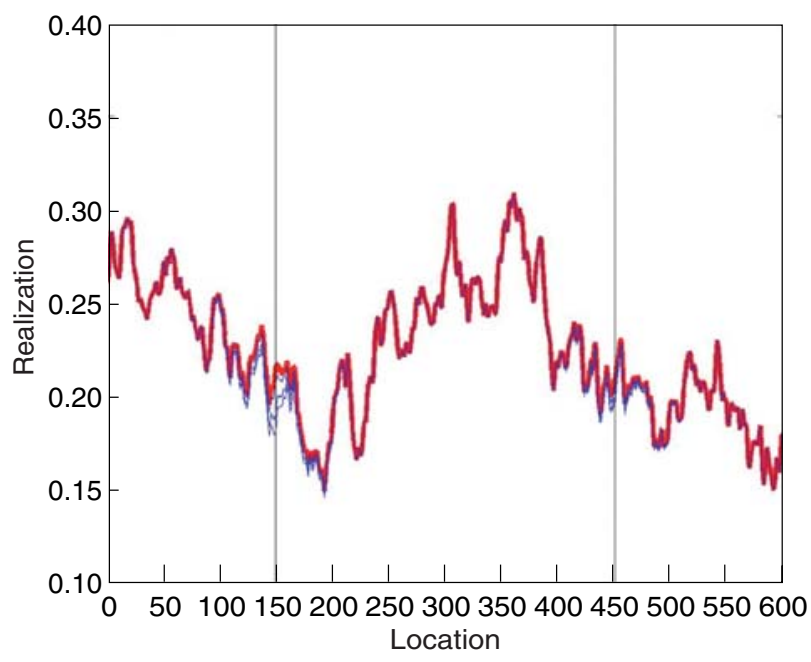

Figure 4

Applying the local gradual deformation method to change the values of points located in 150 and 450 for the same starting one dimensional realization as in Figure 1.

\subsection{Optimization with Gradual Deformation}

The gradual deformation method integrates nicely to optimization processes. Again, this parameterization ensures regularization by narrowing the parameter space: the objective function is reduced to the data mismatch term (Equation 4). The basic flow chart is described in the following subsection. The idea is to calibrate a $\mathbf{y}$ log-permeability field to production data by adjusting the deformation parameters. Instead of considering the $\mathbf{y}$ field, we apply the gradual deformation method to the underlying Gaussian white noise $\mathbf{z}$ for the reason explained in the previous section.

First, a realization chain is built from the initial Gaussian white noise and randomly drawn complementary Gaussian white noises using the gradual deformation method (Equation 8). The resulting Gaussian white noise $\mathbf{z}$ is used to generate a log-permeability field $\mathbf{y}$. This unconditional field can be constrained to the available data referring to conditional kriging (Equation 5). Running a fluid flow simulation for this permeability field provides numerical answers, which are compared to the dynamic data. The quality of the fit is estimated through the objective function. A search process is implemented to determine the deformation parameters minimizing the objective function.

A single realization chain allows for exploring a very tiny part of the realization space and can lead to an unsatisfactory fit. Thus, a new realization chain is constructed by combining the optimal Gaussian white noise identified at the end of the previous search process with a new randomly drawn complementary Gaussian white noise. As explained above, a new 
search process is implemented to investigate this new chain. Sometimes, it may be impossible to determine deformation parameters decreasing the objective function. The reason is that the search process is limited to the chain of realizations built from Equation 8. Of course, this chain is just a very tiny part of the whole search space. Clearly, investigating a given chain does not necessarily lead to a decrease of the objective function. When this happens, we go to another chain: the starting guess is unchanged and a new independent realization is drawn for $\mathbf{z}_{2}$, so that we build a new chain and explore another part of the space. The search process is iterated until an appropriate matching is achieved. Building successively chains of realizations allows for exploring further the realization space. More details can be found in Le Ravalec-Dupin (2005).

Like the pilot point method, the gradual deformation method can be used to identify log-permeability fields from a reduced number of parameters while preserving the spatial variability. It has also other advantages. First, it yields logpermeability fields that depart much more from the prior estimate than the pilot point method. This property is linked to the fact that gradual deformation can be used to modify a whole realization. Second, as long as the gradual combinations are performed with respect to the basic hypotheses (combinations of independent realizations with identical means and covariances), the extreme variations pointed out for the pilot point method are avoided. However, when very local deformations are required to improve the matching of production data, gradual deformation based optimizations may converge very slowly. We propose in the following section to integrate part of the pilot point methodology into the gradual deformation method to make it more efficient in the case of localized deformations.

\section{GRADUAL PILOT POINT METHOD}

\subsection{Optimization with Gradual Pilot Points}

As mentioned above, the pilot point method presents two disadvantages:

- it can attribute non physical values to the pilot points;

- it disregards the possible correlations among the pilot point values.

To bypass the first difficulty, RamaRao et al. (1995) suggested to add inequality constraints to the permeability values of the pilot points.

In this section, we propose an alternative method, named gradual pilot point method, involving the mixing of both pilot point method and gradual deformation method. We aim at keeping as close as possible to the general workflow designed for the pilot point method. The essential difference with the original pilot point method is that pilot points are changed now through gradual deformation rather than optimization.

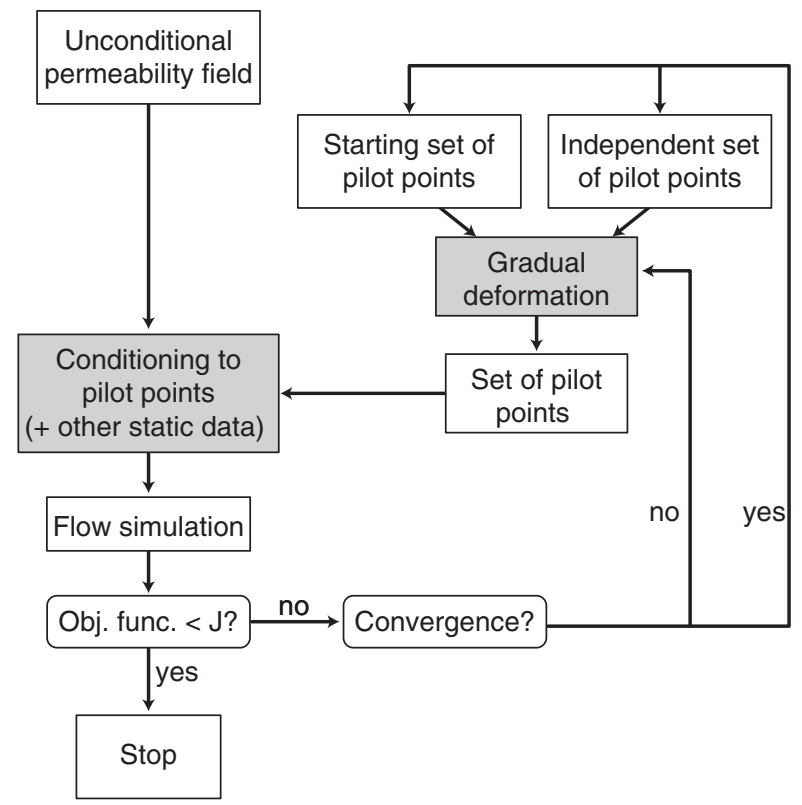

Figure 5

Workflow for a gradual pilot point based optimization.

The workflow of the proposed approach is as follows (Fig. 5). A starting $\mathbf{y}$ log-permeability field is produced from an arbitrary geostatistical simulator. The $\mathbf{y}$ values obtained at the pilot point locations are regarded as the starting pilot point values. Then, we need to draw a complementary set of pilot points. Because the pilot points are located anywhere on the grid, we use the Cholesky decomposition algorithm (e.g., Goovaerts, 1997) to produce an unconditional realization of the pilot point values respecting the same covariance as the log-permeability field. The Cholesky algorithm applies to the covariance matrix of the pilot points. Other geostatistical simulators could be envisioned also as far as they are not restricted to regularly-spaced locations. Using the gradual deformation method to combine the two sets of pilot points provides a set of pilot points, which is used to constrain the starting $\mathbf{y}$ log-permeability field (Equation 5). Then, numerical dynamic answers are simulated and compared to the available dynamic data. By varying the deformation parameters, we build a chain of pilot point realizations. A search process is implemented to identify the gradual deformation parameters leading to the best fit. Thus, we identify a set of optimal pilot points. When the fit is unsatisfactory, the search process is iterated, starting from the set of optimal pilot point. A new complementary set of pilot point values is independently generated. Combining the new starting pilot point values and the new complementary ones provides a new chain, which can be investigated again. 


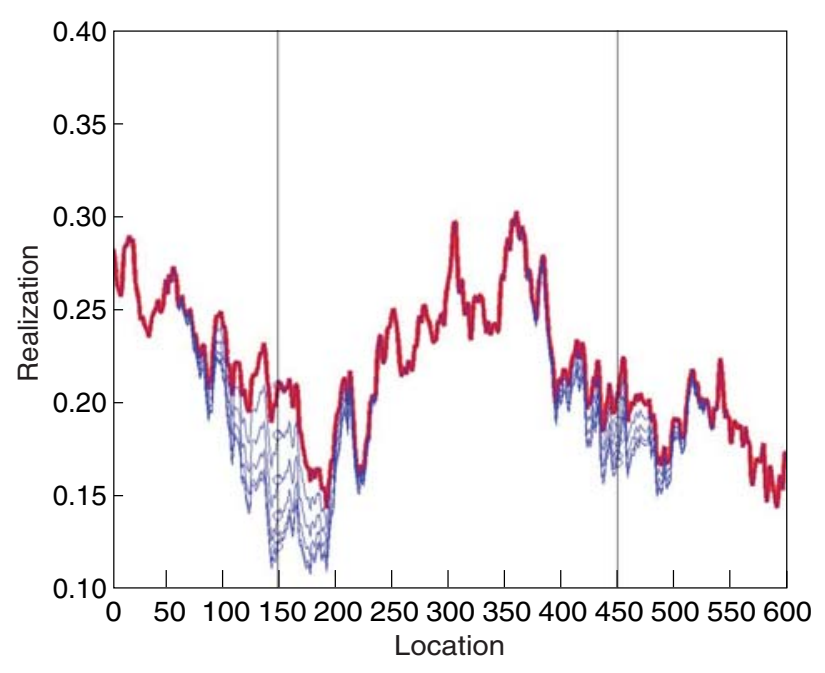

Figure 6

Applying the gradual pilot point method to change the values of points located in 150 and 450 for the same starting one dimensional realization as in Figure 1.

\subsection{Different Gradual Pilot Point Strategies}

A special feature of the proposed approach is that pilot points can be gradually combined from one or several deformation parameters. Two alternatives are distinguished.

\subsubsection{Independent Gradual Pilot Points}

We may apply the local gradual deformation method by independently varying the pilot point values with one deformation parameter per pilot point. In this case, the correlations between the pilot points are disregarded, but the number of degrees of freedom is the same as the one with the original pilot point method.

\subsubsection{Correlated Gradual Pilot Points}

Another possibility is to apply the global gradual deformation rule to modify all pilot point values simultaneously from a single deformation parameter. Thus, the correlations among the pilot points are accounted for, but the number of degrees of freedom is reduced to one, which is less than with the original pilot point method. In this special case, as correlations are accounted for, there is no restriction regarding the number and the location of the gradual pilot points.

\subsubsection{Discussion}

Both approaches have advantages and disadvantages. In any case, whatever the approach selected, we avoid the unreasonable variations noticed when using the original pilot point method, which is a major improvement. An example of deformation obtained by gradually varying pilot point values from a single deformation parameter is shown in Figure 6. The variation range is smaller than with the original pilot point method (Fig. 1), but much more significant than with the gradual deformation method applied at points.

As a result, the gradual pilot point method allows for varying a realization at points from a few parameters while preserving the spatial variability. Unlike the pilot point method, it prevents pilot points from being assigned extreme non physical values and it accounts for the spatial correlations between the pilot points (when a single deformation coefficient is used to modify all of the pilot points). In addition, it
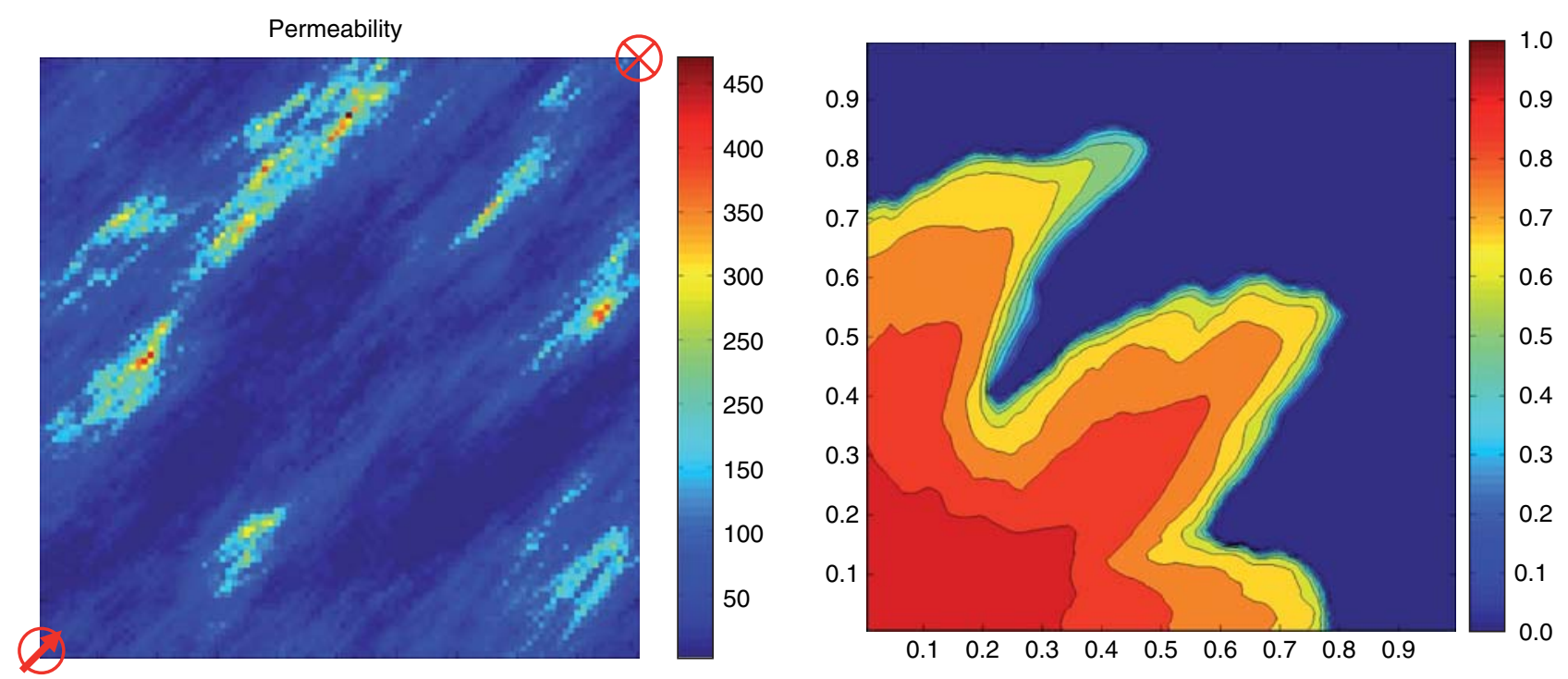

Figure 7

Reference permeability field (left) and reference saturation data (right) collected at time $t$. 

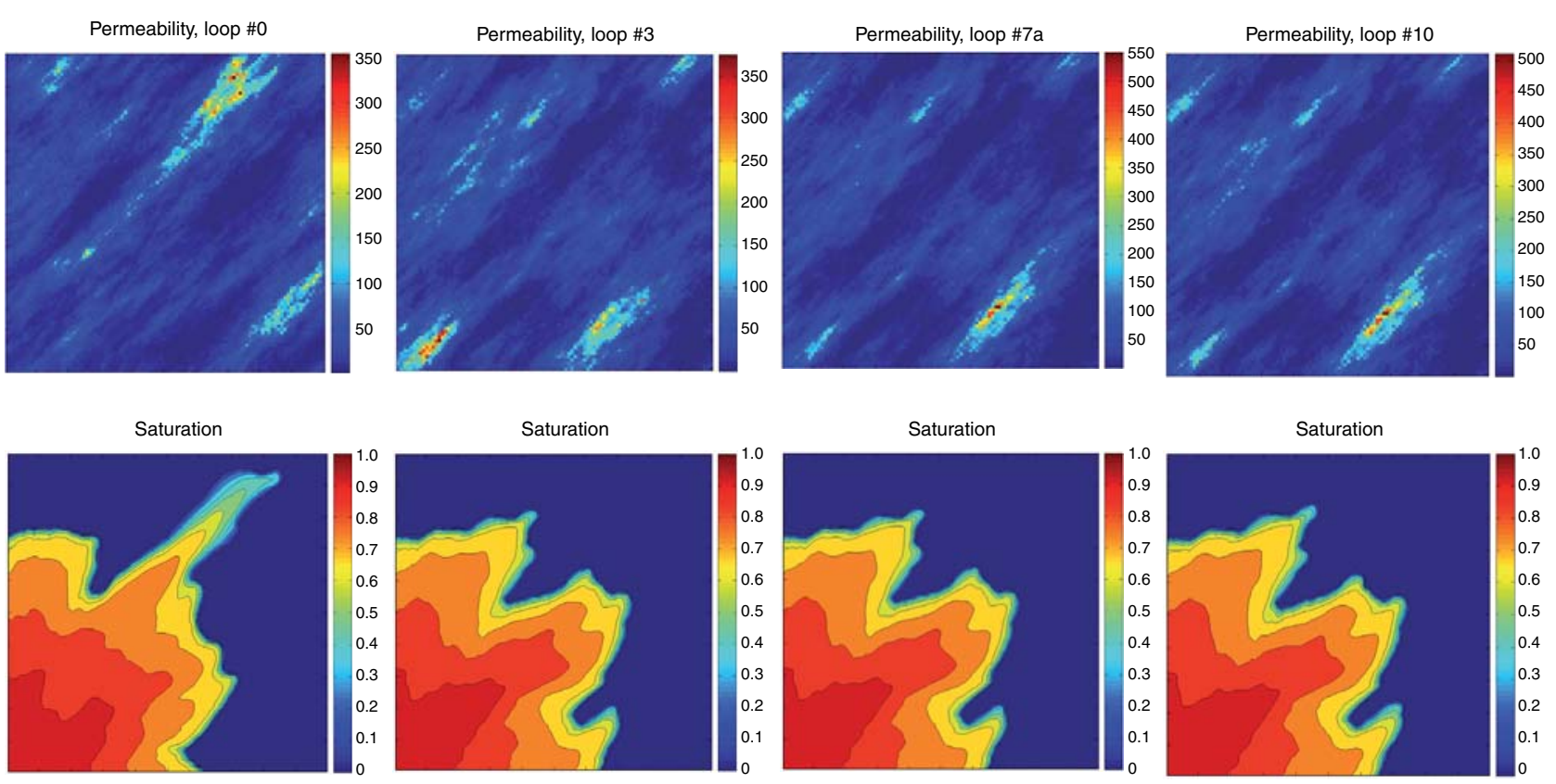

Figure 8

Permeability models (top) and corresponding saturation maps (bottom) at different iterations during the optimization process involving global gradual deformation.

produces more significant deformation than the gradual deformation method when applied to points. This last feature could make it more efficient to reduce the objective function.

\section{APPLICATION TO A SYNTHETIC CASE}

We created a synthetic problem to test the potential of the gradual pilot point method. The proposed problem consists in mapping a permeability field from a saturation map, which is assumed to be deduced from seismic attributes measured at time $t$.

We focus on this example, because it was shown that the global gradual deformation method is not efficient enough to match 4D-seismic attributes around the saturation front (Langlais et al., 2005). Our idea is to use first the global gradual deformation method to approximate the saturation map and second the gradual pilot point method to refine this approximation around the front.

\subsection{Reference Case}

The reference log-permeability field (Fig. 7, left) is discretized over $100 \times 100$ grid cells of size $1 \mathrm{~m} \times 1 \mathrm{~m}$. The log-permeability distribution is normal and characterized by a mean of 3 , a variance of 1 . The variogram is spherical with a correlation length of 60 grid cells along the principle axis at $45^{\circ}$ and 15 grid cells along the perpendicular axis. The reservoir is assumed to be initially saturated by oil. Water is injected in the bottom left corner and produced in the top right corner. Then, a flow simulation is run to estimate the saturation map at time $t$ (Fig. 7, right). This map gives the reference data to be inverted in order to estimate the permeability field if we consider now that this permeability field is unknown.

\subsection{Optimization Experiment}

Given a starting guess (Fig. 8, left), an optimization process involving global gradual deformation was run to minimize the mismatch between the reference saturation map (Fig. 7, right) and the saturation map simulated at time $t$ for the proposed permeability model. Figure 8 shows the successive changes in the permeability model and the resulting saturation maps during optimization. Global gradual deformation induces strong changes in the permeability model mainly during the first iterations. It allows for globally approximating the saturation front. The evolution of the objective function during this first numerical experiment is plotted in Figure 9. It strongly decreases during the first iterations and then, it reaches a plateau. Such a behavior is often evidenced with the gradual deformation method: after a given number of iterations, it gets very difficult to reduce the data misfit. The objective function decreases by $65 \%$ after about 150 fluid flow simulations. Let us note that the number of flow simulations could be significantly reduced if using gradients to guide the minimum search. 


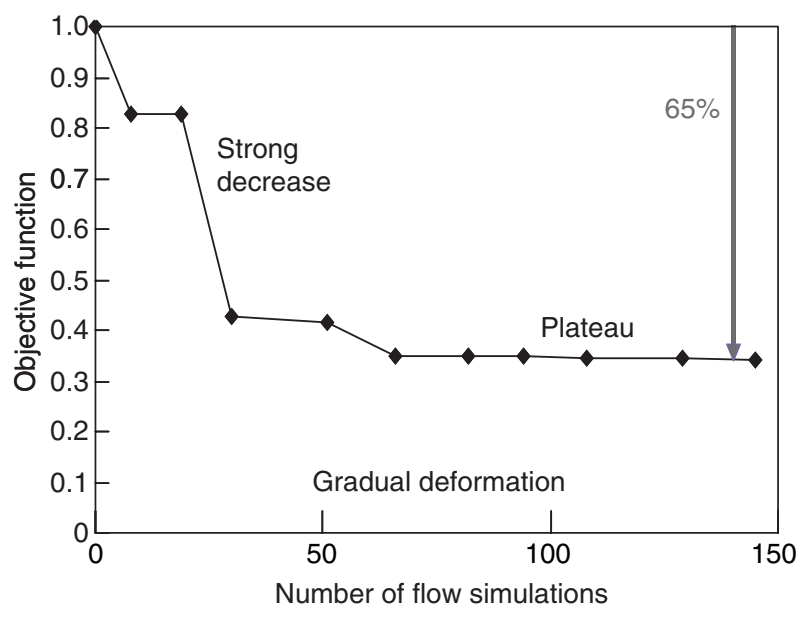

Figure 9

Objective function against the number of flow simulations.

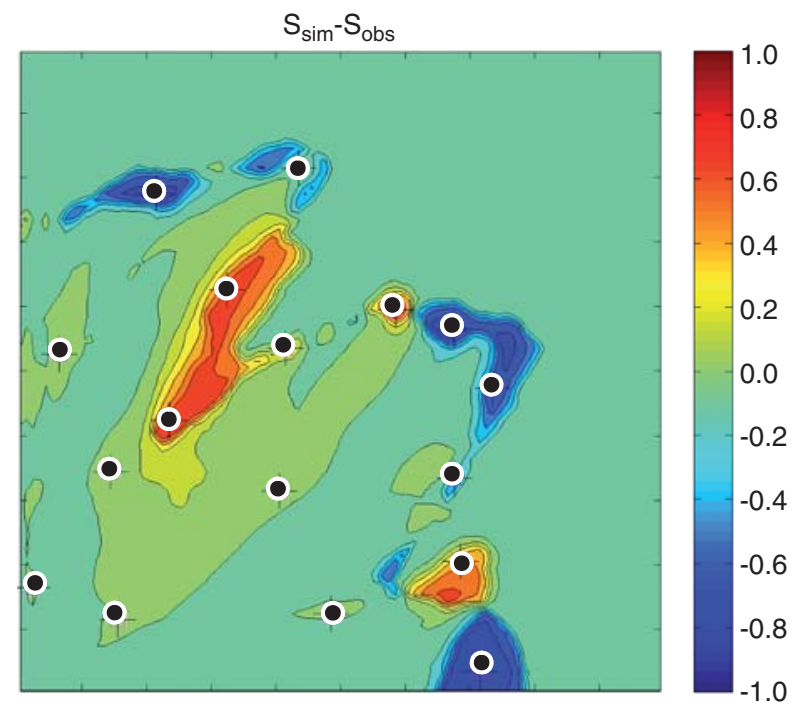

Figure 10

Map of differences between the reference saturation map and the saturation map simulated for the optimal permeability model determined at the end of the first optimization process. Gradual pilot points are located where differences are the largest.

In a second step, we start from the optimal permeability model identified from the previous optimization process and we launch a second optimization with gradual pilot points in order to minimize further the objective function. To locate the gradual pilot points, we compute the differences between the reference saturation map and the one simulated for the previous optimal permeability model. Gradual pilot points are placed where the mismatch is the most important (Fig. 10). All pilot point values are perturbed from the same deformation parameter, which means that spatial correlations are accounted for. The obtained results are displayed in Figure 11. Gradual pilot points provide additional flexibility and make it possible to slightly change the shape of the saturation front. Thus, the objective function is further reduced by $13 \%$ (Fig. 12).

\section{CONCLUSIONS}

The emergence of geostatistics to describe heterogeneous subsurface formations has motivated the development of two geostatistical parameterization techniques: the pilot point method and the gradual deformation method. Basically, they allow for varying a log-permeability (or porosity) field from a few parameters while preserving the spatial variability. These geostatistical parameterization techniques have advantages and disadvantages.

The pilot point method is used to modify only locally the prior permeability estimate. On the other hand, the gradual deformation method provides permeability fields that depart significantly, and not only locally, from the prior estimate.

A few weaknesses have been revealed for the original pilot point method: the pilot points can be attributed unreasonable values and possible correlations between the pilot points are neglected. To bypass these limitations, we introduce the gradual pilot point method. We keep the workflow designed for the pilot point method, but we refer to the gradual deformation method to vary the pilot point values. When the whole set of pilot points is modified at once from a single deformation parameter, pilot point correlations are accounted for, but the number of freedom degrees is 1 . In this case, many pilot points, even close, can be placed on the permeability field. They allow for deforming locally and globally the permeability field. When the pilot point values are modified using one deformation parameter per pilot point, the correlations are disregarded and the number of freedom degrees equals the number of pilot points. In this case, the minimal distance between two pilot points is the correlation length. This technique can be used to produce local deformation only. Whatever the used approach, the unreasonably high or low permeability values are avoided, which is a major improvement compared to the original pilot point method.

A numerical experiment was carried out to underscore the interest of the gradual deformation method and the gradual pilot point method. The problem considered was concerned with the building of a permeability model by matching a saturation map measured at some $t$ time. It was solved using a two-step approach. The gradual deformation method was first used to approximate the saturation front. Then, at some point, it was more and more difficult to improve the matching: the objective function reached a plateau. Therefore, we used the gradual pilot point method, which provided flexibility and allowed for decreasing further the objective function. 

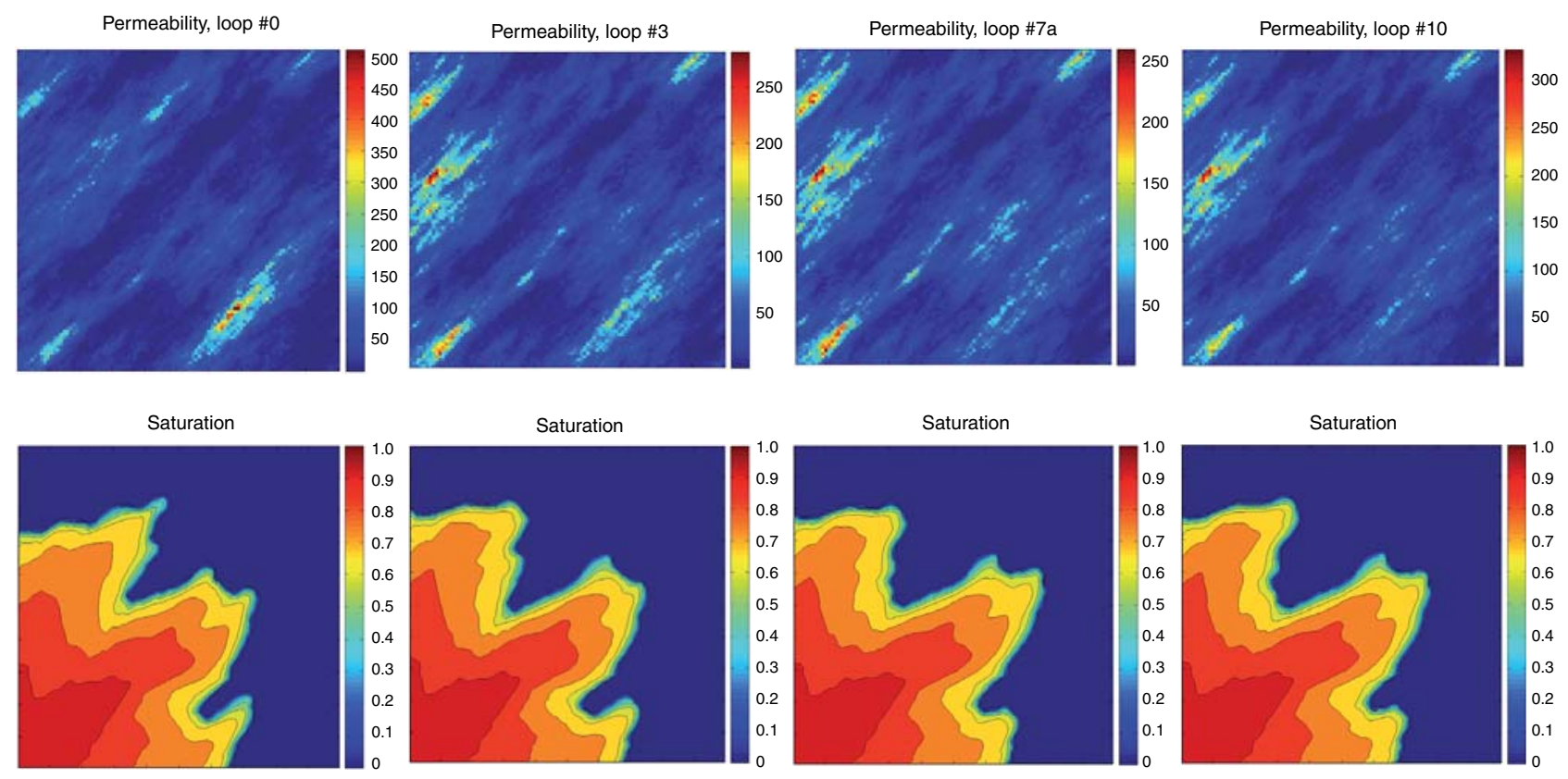

Figure 11

Permeability models (top) and corresponding saturation maps (bottom) at different iterations during the optimization process involving gradual pilot points.

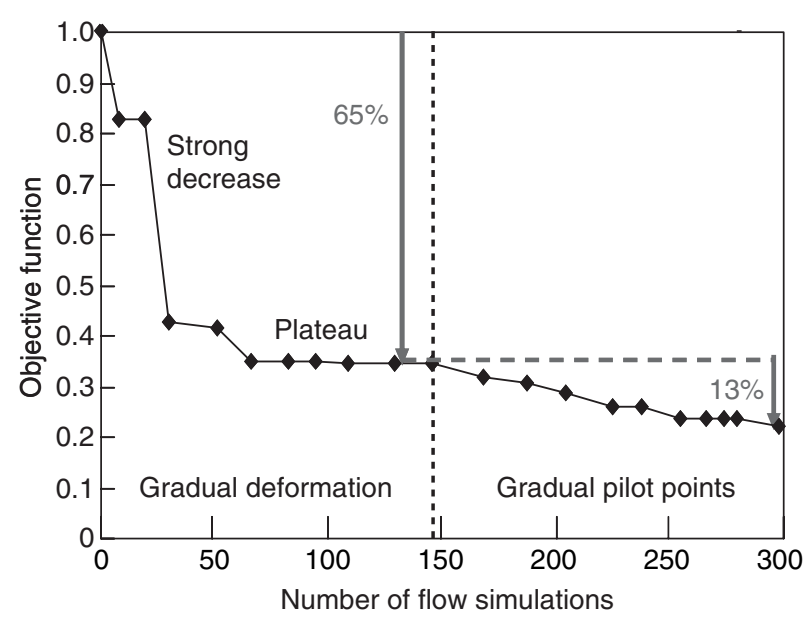

Figure 12

Objective function against the number of flow simulations.

\section{ACKNOWLEDGEMENTS}

This work has been performed within the framework of the CONDOR joint industry project. The authors thank the participating companies for their support: Groupement Berkine, BHP-Billiton, BP, Eni-Agip, Gaz de France, Petrobras and Total.

\section{REFERENCES}

Brac, J., Déquirez, P.Y., Herve, F., Jacques, C., Lailly, P., Richard, V. and Van Nieuh, D.T. (1988) Inversion with a priori information: An approach to integrated stratigraphic interpretation, $58^{\text {th }}$ Ann. Internat. Mtg, SEG, Expanded Abstracts, 841-844.

Chilès, J.-P., and Delfiner, P. (1999) Geostatistics - Modeling spatial uncertainty, Wiley series in Probability and Statistics.

Gomez-Hernandez, J.J., Sahuquillo, A., and Capilla, J.E. (1997) Stochastic simulation of transmissivity fields conditional to both transmissivity and piezometric data. 1. Theory. J. Hydrol., 203, 162-174.

Goovaerts, P. (1997) Geostatistics for natural resources evaluation, Oxford University Press, New York, USA.

Hu, L.Y. (2000a) Gradual deformation and iterative calibration of Gaussian-related stochastic models. Math. Geol., 32, 87-108.

Hu, L.Y. (2000b) Gradual deformation of non-Gaussian stochastic simulations, in Geostatistics 2000 Cape Town, W.J. Kleingeld and O.G. Krige (eds.).

Hu, L.Y., and Le Ravalec-Dupin, M. (2004) An improved gradual deformation method for reconciling random and gradient searches in stochastic optimizations. Math. Geol., 36, 703-719.

Hu, L.Y., Blanc, G., and Notinger, B. (1999) Gradual deformation and iterative calibration of sequential stochastic simulations, 5th Ann. Conf. Int. Ass. Math. Geol., Trondheim, Norway, 6-11 August, 2, 493-498.

Langlais, V., Mezghani, M., Lucet, N., and Huguet, F. (2005) 4D Monitoring of an Underground Gas Storage Case Using an Integrated History Matching Technique, SPE Ann. Tech. Conf. Exhib, SPE 95838

Le Ravalec, M., Notinger, B., and Hu, L.- Y. (2000) The FFT moving average (FFT-MA) generator: an efficient numerical method for generating and conditioning Gaussian simulations. Math. Geol., 32, 701-723. 
Le Ravalec, M., Hu, L.-Y. and Noetinger, B. (2001) Stochastic Reservoir modeling constrained to dynamic data: local calibration and inference of the structural parameters. SPE J., 25-31.

Le Ravalec-Dupin, M. (2005) Inverse stochastic modeling of flow in porous media - Application to reservoir characterization, Editions Technip, ISBN 2-7108-0864-1.

Le Ravalec-Dupin, M., and Hu, L.-Y. (2004) Gradual deformation of boolean simulations, 7th Int. Geostat. Congress, Banff, Alberta, Canada, 26 Sept.-1 Oct.

Le Ravalec-Dupin, M., Notinger, B., Hu, L.-Y., and Blanc, G. (2001) Conditioning to dynamic data: An improved zonation approach. Petrol. Geosci., 7, S9-S16.

Marsily, G. de (1978) De l'identification des systèmes hydrologiques, $P h D$ Thesis, Université Paris VI.

Marsily, G. de, Delhomme, J.P., Coudrain-Ribstein, A., and Lavenue, A.M. (2000) Four decades of inverse problems in hydrogeology, Geophys. Soc. Am., Special paper 348.

Neuman, S.P. (1973) Calibration of distributed parameter groundwater flow models viewed as a multi-objective decision process under uncertainty. Water Resour. Res., 9, 1006-1021.
RamaRao, B.S., LaVenue, A.M., de Marsilly, G., and Marietta, M.G. (1995) Pilot point methodology for automated calibration of an ensemble of conditionally simulated transmissivity fields. 1. Theory and computational experiments. Water Resour. Res., 31, 475-493.

Roggero, F., and Hu, L.-Y. (1998) Gradual deformation of continuous geostatistical models for history matching, SPE Ann. Tech. Conf. Exhib, New Orleans, LA, SPE 49004.

Sun, N.-Z. (1994) Inverse problems in groundwater modeling, Kluwer Acad. Publ., Dordrecht, The Netherlands.

Stallman, R.W. (1956) Numerical analysis of regional water levels to define aquifer hydrology. Trans. AGU, 37, 451-460.

Tarantola, A. (1987) Inverse problem theory - Methods for data fitting and model parameter estimation, Elsevier Science Publishers, Amsterdam, The Netherlands.

Tonellot, T. (1999) Prestack elastic waveform inversion using a priori information, 69th Ann. Internat. Mtg, SEG, Expanded Abstracts.

Final manuscript received in June 2006 